\title{
Optimization strategy of information system for small and medium sized enterprises based on ROI analysis
}

\author{
Fei Zhou \\ Huanggang Normal University, Huangzhou, Hubei, China \\ jsjzhoufei@hgnu.edu.cn
}

\begin{abstract}
Keywords: Information system optimization, ROI, code refactoring, hardware and software classification, dynamic investment.

Abstract. The Small and medium enterprises, which be limited by profitability, management and trade characteristics, are especially sensitive to the cost of information system. In the absence of sufficient financial support and the expected benefits are not clear, optimization of existing information systems, minimize construction and operating costs, is to promote small and medium enterprises to adapt to the development of information technology the best choice. The use of a return on investment (ROI) analysis method, through code reconstruction, hardware and software hierarchical management, dynamic investment and other strategies can reduce enterprise costs and improve the efficiency of information systems.
\end{abstract}

\section{Introduction}

The main difficulty faced by small and medium-sized enterprises in the cost input and operation of information systems is: they have to develop,but the expected benefits are uncertain. The development and popularization of information technology has become the infrastructure of modern enterprise, but any enterprise is hoping to be able to get return for investment through it, and the higher the better, this is also the goal of the enterprise, but it's difficult to analyze and calculate the performance data of information systems in the short run. But there are many mature experience to invest and cost to calculate, through a ROI analysis method, reduce costs can also improve the return on investment, and there are many realistic technology can be achieved.

\section{One of the methods of analysis of ROI for information systems}

The rate of return on investment (ROI) can be simply expressed as Eq. (1).

$$
\text { ROI }=\frac{\text { Cost savings }+ \text { Increased earnings }}{\text { Programme investment }}
$$

The greater the ROI, the better; "Increased earnings" can be quantitative data to measure (such as performance) or non quantitative data to measure (such as brand awareness), it needs long-term observation and comprehensive comparison, and it has certain subjective and uncertainty. It can be used as a reference for subsequent confirmation, we can assume that he is a constant; "Cost savings" and " Programme investment " has relatively more mature experience can be estimated by calculating or obtained. Therefore, the key to improving the return on investment(ROI) is that the investment should be as small as possible, and the cost savings should be as large as possible. In addition, with the development and expansion of enterprise, investment and cost will increase. To be maintaining a relatively stable return on investment, investment growth and cost savings will be Synchronous in a long-term dynamic process. 
An example of a Web system based on fixed investment, when the server performance reaches the upper limit, improving the Web service effect means large-scale bandwidth, server, network equipment and commercial software upgrades, followed by power, maintenance and other upgrade costs. By code refactoring, changing system architecture, configuration of basic services, the Web system can assume greater volume of business, and improve the user experience, improve operational efficiency without increasing investment.

Assuming that the current performance of the Web system hardware and software supports 500,000 people at the same time, the investment is $\mathrm{F}_{50}, \mathrm{ROI}_{50}$ said its return on investment; when the need to support $1,000,000$ people visit, the investment for the $\mathrm{F}_{100}, \mathrm{ROI}_{100}$ said its return on investment. The increase in revenue is assumed to be constant $\mathrm{G}$, the cost savings is assumed to be 0 , the relationship between $\mathrm{F}_{50}$ and $\mathrm{F}_{100}$ is expressed by $\mathrm{n}$. According to experience, investment and Web system performance is not necessarily proportional, but $F_{100}$ compared to $F_{50}$ in practice should be to increase investment, where $\mathrm{n}$ should be greater than 1 . Then:

$$
\begin{aligned}
& \mathrm{F}_{100}=\mathrm{F}_{50} \times \mathrm{n} \\
& \mathrm{ROI}_{100}=\frac{\mathrm{G}}{\mathrm{F}_{100}}=\frac{\mathrm{G}}{\mathrm{F}_{50} \times \mathrm{n}}=\frac{1}{\mathrm{n}} \mathrm{ROI}_{50} \\
& \mathrm{n}>1 \\
& \mathrm{ROI}_{100}<\mathrm{ROI}_{50}
\end{aligned}
$$

The conclusion is that the return on investment $\mathrm{ROI}_{100}$ is less than $\mathrm{ROI}_{50}$.

In the case of no increase in investment, if by using some technical measures to optimize the system, so that the original unit time can be sent to 500,000 people the amount of data to meet $1,000,000$ people. The original need to increase the investment is saved. $\mathrm{F}^{\prime}{ }_{100}$ said the investment which support 1,000,000 people to access the system, ROI' 100 said the corresponding return on investment, then:

$$
\begin{aligned}
& \mathrm{F}_{100}^{\prime}=\mathrm{F}_{50} \\
& \mathrm{ROI}^{\prime}{ }_{100}=\frac{\mathrm{F}_{100} \cdot \mathrm{F}_{50}+\mathrm{G}}{\mathrm{F}^{\prime}{ }_{100}}=\frac{\mathrm{n} \times \mathrm{F}_{50} \cdot \mathrm{F}_{50}+\mathrm{G}}{\mathrm{F}_{50}}=\mathrm{n} \cdot 1+\frac{\mathrm{G}}{\mathrm{F}_{50}}=\mathrm{n}-1+\mathrm{ROI}{ }_{50} \\
& \mathrm{ROI}^{\prime}{ }_{100}>\mathrm{ROI}_{50}
\end{aligned}
$$

Because $\mathrm{n}$ is greater than 1 , the ROI' ${ }_{100}$ is greater than $\mathrm{ROI}_{50}$. That is: the investment to meet the $1,000,000$ people to visit the information system is not increased, but the investment rate of return increased.

\section{Code refactoring Technology}

Code refactoring solves the problem of data storage, computing, and information transfer efficiency. Take the simplest web service as an example, the user can see the same style and same content through the browser with A page or B page. But they are different in fact, as the Table 1 : 
Table 1. Comparative Analysis page A and page B

\begin{tabular}{|c|c|c|}
\hline Web page & Page A & Page B \\
\hline Source Code & $\begin{array}{l}<\text { html }><\text { head }><\text { tit } \\
\text { le }>\text { Hello } \\
\text { World! }<\text { title }></ \text { h } \\
\text { ead }><\text { body }> \\
<\text { p }>\text { Hello } \\
\text { World! }<\text { /p }><\text { /bod } \\
\text { y }></ \text { html }>\end{array}$ & $\begin{array}{l}<\text { html xmlns="http://www.w3.org/1999/xhtml" }> \\
<\text { head }><\text { meta http-equiv="Content-Type" } \\
\text { content="text/html; charset=gb2312" } /><\text { title }>\text { Hello } \\
\text { World! }</ \text { title }><\text { link rel="stylesheet" href="" } \\
\text { type="text/css" }></ \text { head }><\text { body }><\text { p }>\text { Hello } \\
\text { World! }</ \text { p }><\text { p }>\text { \&nbsp;\&nbsp; }</ \text { p }><\text { br }><\text { br }><\text { a } \\
\text { href="'" }></ \mathrm{a}></ \text { body }></ \text { html }>\end{array}$ \\
\hline Result in the browser & Hello World! & Hello World! \\
\hline $\begin{array}{l}\text { The number of characters in } \\
\text { source code(without control } \\
\text { char) }\end{array}$ & 85 & 281 \\
\hline
\end{tabular}

A code and B code show the same effect in most browsers, to achieve the same function. But the amount of B code data is 3.3 times the A code. In other words, if 1,000,000 people are simultaneously accessing the web pages written by B code, the network bandwidth and server performance will be 3.3 times the page written by A code. If the server and bandwidth performance at this time to reach the upper limit, the access quality of more users will be affected without increasing in investment. However, if B code was reconstructed to A code, the same investment to support the maximum number of visits will be 3.3 times the original, that 3,300,000 people can access the A code at the same time. 3,300,000 people and 1,000,000 people for the benefits of the enterprise is clearly different.

Code refactoring is not only a simple method to reduce the amount of data transmission, but also information systems integrated optimization from the whole software level. The main technology is xhtml, xml, ajax, buffer control, code compression, etc., and the goal is to improve the degree of data sharing, reduce data transmission frequency, improve client page interpretation and loading speed, reduce server resource consumption, improve network bandwidth utilization, which can effectively improve the return on investment in information systems.

\section{Hardware and Software Hierarchical Management and Dynamic Investment Strategy}

Small and medium-sized enterprises for information systems investment is limited, as far as possible to achieve the expected return on investment objectives, the need to optimize the portfolio, to avoid large and comprehensive, but should focus on information systems construction and operation of the cost part of the classification analysis, Hierarchical management. For example, for enterprise core business, such as ERP, manufacturing system, financial settlement system, self-built system platform, maintenance and management, to ensure corporate confidentiality and data security; for non-core business, such as portal, customer feedback system, The use of third-party hosting, the server costs, hardware and software maintenance, personnel water and electricity site costs, network bandwidth, information security costs passed on third parties, the enterprise itself only need a single software development design, maintenance and data backup work; Mature public applications, such as business mailboxes, office communications, online sales, can rely entirely on the maturity of the third service platform, the use of common business model, saving development and design costs, and with other competitors are more consistent platform basis, Learning, training, publicity and communication difficulties, improve business efficiency. 
To meet the current development needs of the premise, spend as little money as possible, to build a certain performance and stability of the operation of a period of time the information system is the best choice. If you expect one step in place, engage in large investment, the expected rate of return is not obvious circumstances, the idle resources will increase the cost of enterprises, take up corporate cash flow, drag the development of enterprises; and too frequent investment is not realistic, information systems Design and construction requires time and cost, system deployment, application, training, popularity also takes time and cost. Therefore, the information system investment strategy should be dynamic, but should tend to stage stability.

\section{Conclusions}

Through ROI analysis can be seen in the premise of meeting the needs of information, as far as possible to reduce the information system investment, through code reconstruction and other technologies to save as much as possible, will improve the information system ROI. Hardware and software hierarchical management can invest in scientific combination, effectively reduce the level of enterprise information investment; and maintain a relatively stable dynamic investment strategy will prevent a one-time investment is too large and reduce the incidental costs, and the return on investment will maintain a more stable level or Have further room for improvement.

\section{References}

[1] Sen Liu. Analysis on Return on Investment of Enterprise Cloud Computing Information. China Science and Technology Forum. June 2014.

[2] Jie Xu, Rui Yao. Enterprise investment cost monitoring algorithm based on cloud computing[J]. Computer Engineering and Science. 2014, 36(7).

[3] Cheng Wang, Shaoyuan Li, Lixiao Zheng, Jin Hou, Meiqin Zeng, Huimin Liu. Web front - end performance optimization scheme and practice. Computer Applications and Software. 2014(12).

[4] Xueyang Zhang, Haipeng Yu, Web - based Application System Front - End Page Performance Optimization. Fujian computer. May 2012. 